ze über einen längeren Zeitraum. Die Steigerungsraten waren fast überall nur mäßig oder die Steuersätze der neu eingeführten $\mathrm{CO}_{2}$ - und/ oder Energiesteuern blieben über Jahre hinweg konstant. Ebenso wurden klimapolitisch motivierte Steuererhöhungen teilweise durch Senkungen bei anderen Energiesteuern kompensiert. Insgesamt bestehen bei der Höhe und Steigerungsrate der Steuersätze also in ganz Europa noch Defizite.

\section{Möglichkeiten einer Weiterentwick- lung auf EU-Ebene}

Nachdem die bisherigen Reformschritte alle mehr oder weniger unkoordiniert als ,nationale Alleingänge" stattgefunden haben, stellt sich die Frage, ob es nicht auf EU-Ebene Möglichkeiten eines weitergehenden, koordinierten Vorgehens gibt. Die politischen Aussichten hierfür sind jedoch gegenwärtig schlecht. Die Kommissionsvorschläge von 1992 und 1995, eine EU-weite $\mathrm{CO}_{2}$-/Energiesteuer einzuführen, sind gescheitert, und der nochmals abgeschwächte aktuelle Kommissionsvorschlag, der im wesentlichen die Erhöhung der bestehenden EU-Mindeststeuersätze und die Einführung von Mindestsätzen für Erdgas, Kohle und elektrischen Strom im Jahr 2002 vorsieht, steckt seit 1997 fest. Der wesentliche Anstoß für die Weiterführung der ökologischen Steuerreform wird also nach wie vor von den Einzelstaaten ausgehen müssen, wobei Deutschland als wirtschaftlich bedeutendster Staat der EU durch eine systematischere und konsequentere Fortführung der ökologischen Steuerreform ein besonders wichtiges politisches Signal für die Mitgliedstaaten setzen könnte.

\section{Literatur}

Bach, S.; Kohlhaas, M.; Linscheidt, B.; Seidel, B.; Truger, A., 1999: Ökologische Steuerreform: Wie die Steuerpolitik Umwelt und Marktwirtschaft versöhnen kann, Berlin.

Deutsches Institut für Wirtschaftsforschung / Finanzwissenschaftliches Forschungsinstitut an der Universität zu Köln [DIW/FiFo], 1999: Anforderungen an und Anknüpfungspunkte für eine Reform des Steuersystems unter ökologischen Aspekten, Berichte des Umweltbundesamtes 99/3, Berlin.

Ewringmann, D.; Linscheidt, B.; Truger, A., 1996: Nationale Energiebesteuerung: Ausgestaltung und Aufkommensverwendung, Finanzwissenschaftliche Diskussionsbeiträge Nr. 96-1, Köln.
Linscheidt, B.; Truger, A., 1995: Ökologische Steuerreformvorschläge vor dem Hintergrund des bestehenden Steuersystems, Berlin.

Linscheidt, B.; Truger, A., 2000a: Ökologische Steuerreform: Ein Plädoyer für die Stärkung der Lenkungsanreize. In: Wirtschaftsdienst 2/2000, S. $98 \mathrm{ff}$.

Linscheidt, B.; Truger, A., 2000b: Energiebesteuerung und Sonderregelungen für die Industrie - Ein Konzept prozessspezifischer Freibeträge, in: Zeitschrift für angewandte Umweltforschung, Jg. 13, Heft 1/2 2000, S. 50 ff.

Truger, A., 2000: Ökologische Steuerreformen in Europa - wo steht Deutschland?, WSI-Diskussionspapier Nr. 87, Juni 2000.

\section{Kontakt}

Dr. Achim Truger

WSI in der Hans-Böckler-Stiftung

Bertha-von-Suttner-Platz 1, D-40227 Düsseldorf

Tel.: + 49 (0) $211-7778-264$

Fax: + 49 (0) $211-7778-190$

E-Mail: Achim-Truger@BOECKLER.DE

\title{
Die Zukunft des Internet - aus der Sicht internatio- naler Experten
}

\author{
von Klaus Beck, Universität Erfurt
}

Das Internet hat Zukunft - darüber besteht weitgehend Einigkeit. Wie diese Zukunft allerdings aussieht, ist eine offene Frage. Die Prognosen über Wachstum, Nutzung und Nutzen des Internet gehen ebenso weit auseinander wie die Hoffnungen und $\mathrm{Be}-$ fürchtungen, die sich mit Computernetzen verbinden. Auf den ersten Blick scheint es so viele Prognosen wie Experten zu geben. Die Vielfalt der Meinungen und Einschätzungen - an sich ein erfreuliches Zeichen für einen lebendigen öffentlichen Diskurs sollte durch die Studie über den „Computer als Medium der Medienintegration" nicht um eine weitere Prognose bereichert werden. Es ging vielmehr darum, zwischen den verschiedenen Positionen zu vermitteln und die Einschätzungen diskursiv - mit Hilfe eines weiterentwickelten Delphi-Verfahrens - zu validieren. 
$\mathrm{Zu}$ diesem Zweck haben der Lehrstuhl für Kommunikationswissenschaft an der Universität Erfurt und die Unternehmens- und Technologieberatung Booz, Allen \& Hamilton gemeinsam ein internationales Forschungsprojekt durchgeführt, das vom Bundesministerium für Bildung, Wissenschaft, Forschung und Technologie sowie vom Bundesministerium für Wirtschaft und Technologie gefördert wurde. ${ }^{1}$ Weil Computernetze, insbesondere das Internet immer weitere Bereiche des Alltags privater Nutzer wie geschäftlicher Anwender durchdringen, wurde die Untersuchung thematisch sehr breit angelegt. Für die Expertenbefragung wurden sechs verschiedene Fragebogenmodule entwickelt, und zwar zu den Themenkreisen:

- Allgemeine Entwicklungen (Technik, Infrastruktur, Kommerzialisierung, Segmentierung und Globalisierung der computervermittelten Kommunikation)

- Information, Unterhaltung und Spiele (Medien und Computerspiele)

- Virtuelle Beziehungen und Cybersex

- Electronic Commerce (Business-toconsumer: Shopping, Banking, Booking)

- $\quad$ Arbeitswelt (Telearbeit)

- Lehren und Lernen (Allgemeinbildung).

Die einzelnen Fragebogenmodule umfassten je nach Komplexität der Themenstellung zwischen 18 und 25 Fragen, für deren Beantwortung ca. 35 Minuten benötigt wurden. Bei allen Fragestellungen ging es ausdrücklich nicht darum, was technisch machbar sein wird, sondern wann welche Technik auf welche Art und Weise angewandt werden wird. Unser Interesse galt also der Veralltäglichung der Technik, der breiten gesellschaftlichen Akzeptanz und Diffusion der Computernetze sowie möglicher Folgen.

Alle Teilnehmer erhielten das Fragebogenmodul über die „Allgemeinen Entwicklungen" sowie - je nach ihrer persönlichen Expertise - bis zu drei weitere fachbezogene Fragebogenmodule. Von allen Fragebögen wurden deutsch- und englischsprachige Versionen erstellt; alternativ konnten die von uns angeschriebenen Experten die Fragebögen auch als password-geschützte WWW-Dokumente ausfüllen. Die Online-Alternative wurde insbesondere von den Teilnehmern aus Asien und Ozeanien sowie den Experten aus der Industrie genutzt, so dass sich die Entwicklung dieses zusätzlichen Erhebungsinstruments bewährt hat. Den Befragten wurden kurze Statements (einzelne „Visionen“) vorgelegt, zu denen sie Stellung nehmen sollten. Gefragt wurde entweder nach dem Grad ihrer Zustimmung (4erSkala), nach dem Zeitpunkt des Eintretens einer Vision (3 Intervalle: bis 2005, -2010, -2015, sowie ,später“ und „nie“), nach der Quantifizierung (4er-Skalen) oder der Quantifizierung im Zeitverlauf. Erhoben wurden ferner soziodemographische Daten und - wie bei der Delphi-Methode üblich - die Selbsteinschätzung der persönlichen Expertise (4erSkala).

Bis zum September 1998 wurden insgesamt 2.014 Experten um ihre Teilnahme gebeten, sie erhielten insgesamt 4.482 Fragebogenmodule. 480 Personen beteiligten sich an der ersten Befragungsrunde (Rücklaufquote: $23,8 \%$ ), davon $18,1 \%$ online. Für die erste Auswertung lagen 1.083 Fragebogenmodule vor. ${ }^{2}$ Die aggregierten Ergebnisse der ersten Befragungsrunde wurden in die Fragebögen für die 2. Delphi-Runde integriert, damit die Teilnehmer vor dem Hintergrund des anonymisierten Gruppenurteils ihre Prognosen überdenken und ggf. korrigieren konnten. An der zweiten Befragungsrunde, die im Winter 1998/1999 durchgeführt wurde, beteiligten sich 360 Experten (Rücklaufquote: $75 \%$ ) mit insgesamt 765 Fragebogenmodulen. 51,2\% der Teilnehmer waren Wissenschaftler, 28,8\% Entscheider aus der Wirtschaft und 20\% Vertreter von Interessengruppen (Verbraucherschutz, Gewerkschaften etc.). Über drei Viertel $(76,7 \%)$ der Befragten leben und arbeiten in Deutschland, weitere 4,2\% in Europa. Jeder Zehnte stammte aus den USA oder Kanada; $7 \%$ aus Asien und 2,4\% aus Australien. ${ }^{3}$ Trotz des Übergewichts der deutschen Experten, erlaubt es die Zusammensetzung des Panels durch die Kontrastierung mit der ausländischen „Kontrollgruppe“, ein ggf. abweichendes Prognoseverhalten zu erkennen. ${ }^{4}$

Aus der Fülle der Ergebnisse können hier nur einige Kernaussagen vorgestellt werden. Demnach erweisen sich viele populäre Utopien - mögen sie auch von Protagonisten (und Propagandisten) der Netzentwicklung wie Gates, Negroponte oder Dertouzos stammen - als überzogen. Der heutige Betrachter wird die 
„Informationsgesellschaft“ des Jahres 2010 trotz der „Medienrevolution“ durchaus noch wiedererkennen, so jedenfalls die ganz überwiegende Einschätzung der von uns Befragten. Weniger das Tempo der Veränderung, als vielmehr die Breite der betroffenen Gesellschafts- und Alltagsbereiche sowie die Tiefe der Strukturveränderungen prägen die Sicht der internationalen Experten. So bilden sich zum Beispiel folgende Tendenzen heraus:

- $\quad$ Bis zum Jahr 2010 werden etwa 25 bis 40 Prozent der privaten Haushalte in den entwickelten Industriestaaten über einen universellen Zugang zu digitalen Medien und Kommunikationsdiensten verfügen, den sie regelmäßig nutzen.

- Die Nutzung der Computernetze wird mit Hilfe unterschiedlicher Endgeräte erfolgen - die ,universelle Kommunikationsmaschine" wird also allenfalls eine digitale, vielleicht TCP/IP-basierte Infrastruktur sein, nicht jedoch die Gestalt eines einheitlichen Interface annehmen.

- $\quad$ Ein Haupttrend liegt in der Entwicklung und Verbreitung mobiler Kommunikationsgeräte; das Handy hat demnach gute Chancen, zusätzliche Funktionalitäten zu gewinnen.

- Die bekannten Modi der Mediennutzung (Unterhaltung, Information, Kommunikation) werden in ihren Grundzügen erhalten bleiben; auf der Angebotsseite kommt es $\mathrm{zu}$ einer Kommerzialisierung und Segmentierung. Die Trennung von redaktionellem Inhalt, PR und Werbung wird weiter erodieren, zumal auch Markenartikler und andere Unternehmen selbst zu Anbietern von Medieninhalten werden.

- Die direkte Kommunikation (Face-to-face) wird ihre sehr hohe soziale Bedeutung behalten, E-Mail wird Teile des Telefon- und Briefverkehrs substituieren. Im übrigen werden computervermittelte Kommunikationsformen als zusätzliche Chance für die Erweiterung des persönlichen sozialen Netzwerks und die Teilhabe in virtuellen Gemeinschaften global genutzt.

- Ausschlaggebend für die Wahl eines bestimmten Mediums bzw. eines Kommunikationsmodus bleibt - trotz erweiterter Wahlmöglichkeiten - der Beziehungsaspekt: Entscheidend ist, mit wem (Partner,
Familie, Freunde, Bekannte oder Fremde) man kommunizieren will, und weniger, welcher Anlass oder Zweck (Task orientation) damit verfolgt wird.

- $\quad$ Bildung, Einkommen und Lebensstil (z.B. großstädtische, erwerbstätige Singles) bleiben auch in den nächsten 10 bis 15 Jahren prägende Kriterien für die regelmäßige Online-Nutzung. Die „Digital divide" könnte also zu einem nachhaltigen Problem unserer Gesellschaft werden.

- Im Bildungswesen trägt der Prozess der Medienintegration zur Entwicklung eines spezialisierten Bildungsnetzes bei, ohne dass es mittelfristig zu strukturellen Umwälzungen im Institutionen-Gefüge der Allgemeinbildung (Schulen und Hochschulen) kommen wird. Die virtuelle Universität wird im nächsten Jahrzehnt nicht zum Regelfall.

- Wachsende Wissensklüfte, soziale Ungleichheit und divergierende Medienkompetenzen begleiten zumindest mittelfristig den Prozess der Medienintegration. Dies gilt nicht nur im globalen Maßstab, sondern auch für entwickelte Industrie- bzw. „Informationsgesellschaften“.

- Im wissenschaftlichen Publikations- und Bibliothekswesen ermöglicht die Medienintegration einschneidende Veränderungen: Bibliotheken werden ihr Dienstleistungsangebot erweitern und elektronische Texte (preprints) werden den wissenschaftlichen Diskurs sowie den Wissenstransfer verändern. Prognostiziert wird u.a., dass Wissenschaftler noch unabgeschlossene Publikationen im Netz zur Diskussion stellen und der Scientific community die Möglichkeit geben, an diesen Texten weiter zu schreiben. Ein solches „Ende des Autors“ könnte weitreichende Folgen auf das gesamte Reputationswesen der Wissenschaft zeitigen.

- Electronic-Commerce ist das Epizentrum der Entwicklungen zur „Informationsgesellschaft", wobei einzelne Branchen sehr unterschiedlich betroffen sind. Marketing und Vertrieb sowie die gesamte Wertschöpfungskette unterliegen einem strukturellen Wandel. Vor allem ,immaterielle“ Güter wie Software und Musik, aber auch Bücher und EDV-Hardware werden in 
deutlich höherem Maße online verkauft werden als beispielsweise Lebensmittel, Kleidung, Möbel oder Autos.

- Im Business-to-consumer-Sektor erweisen sich E-Banking und E-Booking als Vorreiter; das Filialgeschäft der Banken und die Reisebüros werden hiervon in sehr hohem Maße betroffen sein. Zu den Verlierern werden außerdem die Videotheken gehören, die sich gegen digitale Video-ondemand-Angebote nicht mehr lange werden behaupten können.

- Kurz- und mittelfristig sind leicht negative Beschäftigungseffekte zu erwarten (NettoArbeitsplatz-Bilanz), zunächst vor allem in den Industriestaaten.

- Alternierende Telearbeit wird primär von geringer Qualifizierten im Dienstleistungssektor geleistet; die Erwerbsverhältnisse werden sich durch- und tiefgehend wandeln. Feste, arbeitsrechtlich besonders geschützte Beschäftigungsverhältnisse werden mehr und mehr projektbezogenen Kooperationen weichen. Erwerbstätige werden als „Selbstbeschäftiger“ vorübergehender „Teil“ oder Partner virtueller Unternehmen.

Einige Ergebnisse der Delphi-Studie unterscheiden sich von denen vieler anderer Prognosen durch eine gewisse Nüchternheit, die manche als Mangel an Vision empfinden mögen. Besonders deutlich wird das an zwei viel diskutierten Themenkomplexen: der Konvergenz von Personal Computer und Fernsehen sowie der Arbeitsmarktbilanz.

Unsere Untersuchungen legen nämlich den Schluss nahe, dass auch 2010 nicht die hoch integrierte Kommunikationsmaschine den Alltag prägen wird. Spracherkennung und Steuerungssysteme sowie dreidimensionale grafische Benutzeroberflächen setzen sich vermutlich erst in den Jahren nach 2010 durch. Eine erweiterte TV-Fernbedienung erlaubt zwar schon bald die individualisierte und selektive Nutzung von Informations- und Unterhaltungsangeboten, doch lassen sich auch im Jahr 2010 noch zwei typische Mediennutzungssettings unterscheiden: Die unterhaltungsorientierte Mediennutzung gleicht im Grunde der heutigen Fernsehnutzung. Interaktive Eingriffe in die narrative Dramaturgie oder die Partizipation an Game-Shows sind wenig verbreitete
Ausnahmen; weiter ausdifferenziert hat sich hingegen das zielgruppenbezogene Angebot im Sinne des „Narrow-casting“. Gleichwohl erfreuen sich die großen Unterhaltungs-Events, insbesondere Sportübertragungen, Shows und Spielfilme weiterhin hoher Einschaltquoten. ${ }^{5}$

Das bedeutet zum Beispiel: Auf absehbare Zeit werden sich trotz der - begrenzten - Zunahme von Special Interest-Programmen die Fernsehvollprogramme erhalten. Die Auflösung jeglicher Senderloyalität ist ebenso unwahrscheinlich wie der vollständige Zerfall der Öffentlichkeit.

Ebenso ernüchternd wirkt das Ergebnis, das die Beschäftigungseffekte der „Informationsgesellschaft" kurz- und mittelfristig „leicht negativ" sein werden. ${ }^{6}$ Das widerspricht dem Pflichtpensum an Optimismus, der auf Messen wie der Cebit und der Internationalen Funkausstellung versprüht zu werden pflegt. Es ist aber durchaus plausibel, wenn man die euphorischen Prognosen über die Entwicklung des Electronic Commerce ernst nimmt: Wo der Kontakt zwischen Leistungserbringern und Leistungsnutzern übers Netz erfolgt, werden traditionelle Zwischenstufen wie Vertrieb und Handel ausgeschaltet. Electronic Banking wird also zu einer Ausdünnung der Filialstruktur der Banken führen, elektronischer Buchvertrieb wird den traditionellen Buchhandel treffen, Electronic Shopping alte Intermediäre schwächen. Dies stellt zwar keine Globalaussage über die weltweite Arbeitsplatzbilanz der neuen Online-Welt dar. Schwellenländer mit günstigen Entlohnungsstrukturen mögen mittelfristig von der Entwicklung profitieren, auch ist der Gang der Dinge ,in the long run“ nicht übersehbar. Für die nächsten Jahre aber sehen unsere Experten keinen Nettozuwachs von Arbeitsplätzen. Was das bei der hohen Sockelarbeitslosigkeit in vielen europäischen Gesellschaften bedeutet, wird in dieser empirischen Studie nicht über Gebühr ausgebreitet. Es ist aber unübersehbar.

\section{Anmerkungen}

1) Förderkennzeichen 01BN801/9

2) Für detaillierte Angaben zur Zusammensetzung des Ausgangs-Panels sowie des Rücklaufs der 1. Befragungsrunde nach Weltregion und Expertengruppe vgl. Beck, Glotz, Vogelsang 2000, S. 27-36 
3) Vgl. Beck, Glotz, Vogelsang 2000, S. 37-46

4) Bemerkenswerte Unterschiede gab es vor allem bei den Prognosen zu Lehren und Lernen mit Computernetzen.

5) Vgl. Beck, Glotz, Vogelsang 2000, S. 67-70

6) Vgl. Beck, Glotz, Vogelsang 2000, S. 58-60

\section{Bibliographische Angaben}

Beck, Klaus; Glotz, Peter; Vogelsang, Gregor, 2000: Die Zukunft des Internet. Internationale Delphi-Befragung zur Entwicklung der OnlineKommunikation. Konstanz: uvk Medien

\section{Kontakt}

Dr. Klaus Beck

Institut für Kommunikations- und

Medienwissenschaft

Universität Leipzig

Klostergasse 5, D-04109 Leipzig

E-Mail: klaus.beck@uni-erfurt.de

\section{$\gg$}

\section{Internationale Experten- befragung $z u$,Future Competitiveness“}

\author{
von H. Hungenberg, St. Lackner, T. Wulf, \\ Friedrich-Alexander-Universität Erlagen- \\ Nürnberg, und D. Pommerening, Handels- \\ hochschule Leipzig
}

Deutschland kann sich im internationalen Standortwettbewerb um die Ansiedlung sog. „Zukunftsindustrien“ sehr gut behaupten und seine Position sogar festigen - das ist eines der Ergebnisse einer internationalen Expertenbefragung, die an der Handelshochschule Leipzig und an der FriedrichAlexander-Universität Erlangen-Nürnberg durchgeführt wurde. Insgesamt wurden 257 Politiker, Spitzenmanager und Professoren aus 34 Ländern nach ihrer Einschätzung der zukünftigen Wettbewerbsfähigkeit ausgewählter Nationen und des Wachstumspotentials bestimmter „Zukunftsbranchen“ detailliert befragt.

Im Zeitalter einer fortschreitenden Globalisierung und eines sich beschleunigenden technischen Wandels verlieren - nach Meinung vieler
Experten - die Industrieländer sukzessive ihre Wettbewerbsfähigkeit in Branchen wie z. B. der Chemie- und Elektroindustrie, die in den letzten zwanzig Jahren die wirtschaftliche Entwicklung ganz wesentlich geprägt haben. Die Zukunft wird nicht mehr bestimmt durch eine herausragende Stellung in einzelnen Technologiebereichen, sondern durch die Fähigkeit, vielfältige Informationen und spezifisches Wissen $\mathrm{zu}$ generieren und nutzenorientiert anzuwenden. Dementsprechend gehen viele Experten davon aus, dass sog. wissensbasierte Branchen die Spitzenbranchen der Zukunft sein werden. Welche Industrien zum Kreis dieser wissensbasierten Branchen zählen und welchen Ländern die größten Potentiale für diese Branchen zugeschrieben werden - das sollte in dieser Befragung ermittelt werden.

\section{Zukünftige Wettbewerbsfähigkeit von Nati- onen}

Hinsichtlich der Einschätzung der zukünftigen Wettbewerbsfähigkeit zeigen die Ergebnisse der Untersuchung eine eindeutige Rangfolge der zehn analysierten Länder: Die USA besitzen das größte Potential im Bereich der wissensbasierten Branchen, gefolgt von Großbritannien und Deutschland. Ein vereintes Europa kommt nahe heran an die als Einzelland dominierenden USA. Japan ist eher mittelklassig einzuordnen und die ,schlafenden Riesen“ China, Indien und Russland bleiben auch in Zukunft, was sie sind - nämlich schlafende Riesen (vgl. Abb. 1).

Diese Untersuchung bestätigt damit den dramatischen Wandel, der in den letzten 10 15 Jahren in der Weltwirtschaft stattgefunden hat: Noch in der zweiten Hälfte der 80er Jahre galt die Wirtschaft der USA als rückständig. „Japan Inc.“ wurde als das zukunftsträchtige Erfolgsmodell gepriesen. In Europa beobachtete man mit Sorge die Abwanderung von industriellen Großinvestitionen in die Schwellenländer, und speziell in Deutschland befasste man sich seit 1990 überwiegend mit den Problemen der Wiedervereinigung. Ganze Industriesegmente wie die Unterhaltungselektronik oder der Großschiffbau existierten in den USA und Europa praktisch nicht mehr, kurz: Die Angst um die Zukunftsfähigkeit der alten Industrienationen griff um sich. 